\title{
LESION TARGETED ULTRASONOGRAPHY GUIDED ABDOMINAL WALL ANESTHESIA FOR INCISIONAL HERNIA REPAIR IN ACCORDANCE WITH ERAS PROTOCOL
}

\author{
Tayfun Aydın and Onur Balaban
}

\author{
M.D. Associate Professor. Kutahya Health Sciences University Hospital, Department of Anesthesiology and \\ Pain, Kutahya, Turkey.
}

\begin{abstract}
Background: Enhanced recovery after surgery (ERAS) protocols should incorporate multimodal analgesic usage and minimizing opioids with regional anesthesia techniques to improve pain control. Abdominal trunk blocks are highly recommended in ERAS guidelines for significant reduction of pain intensity and opioid consumption (Figure 1). We present a case of incisional hernia repair which was operated under combination of rectus sheet block and subcostal transvers abdominis plane (TAP) block. The block combination provided sufficient surgical anesthesia and postoperative analgesia with early mobilization and early discharge.
\end{abstract}

Methods: The patient was 78-year-old male who was scheduled for incisional hernia repair. His medical history included hypertension, diabetes mellitus, coronary artery disease and chronic obstructive pulmonary disease. According to American Society of Anesthesiology (ASA) risk classification, the patient was evaluated as ASA III risk class.

Even an epidural was high risk like general anesthesia, we decided to perform lesion targeted abdominal wall anesthesia for the operation to be in accordance with ERAS protocols. After ultrasonographic examination of the hernia, rectus sheets surrounding hernia borders were blocked under ultrasound guidance. We concluded to add subcostal TAP block in order to strengthen the anesthetic effect of rectus sheet block and warrant a full surgical anesthesia.
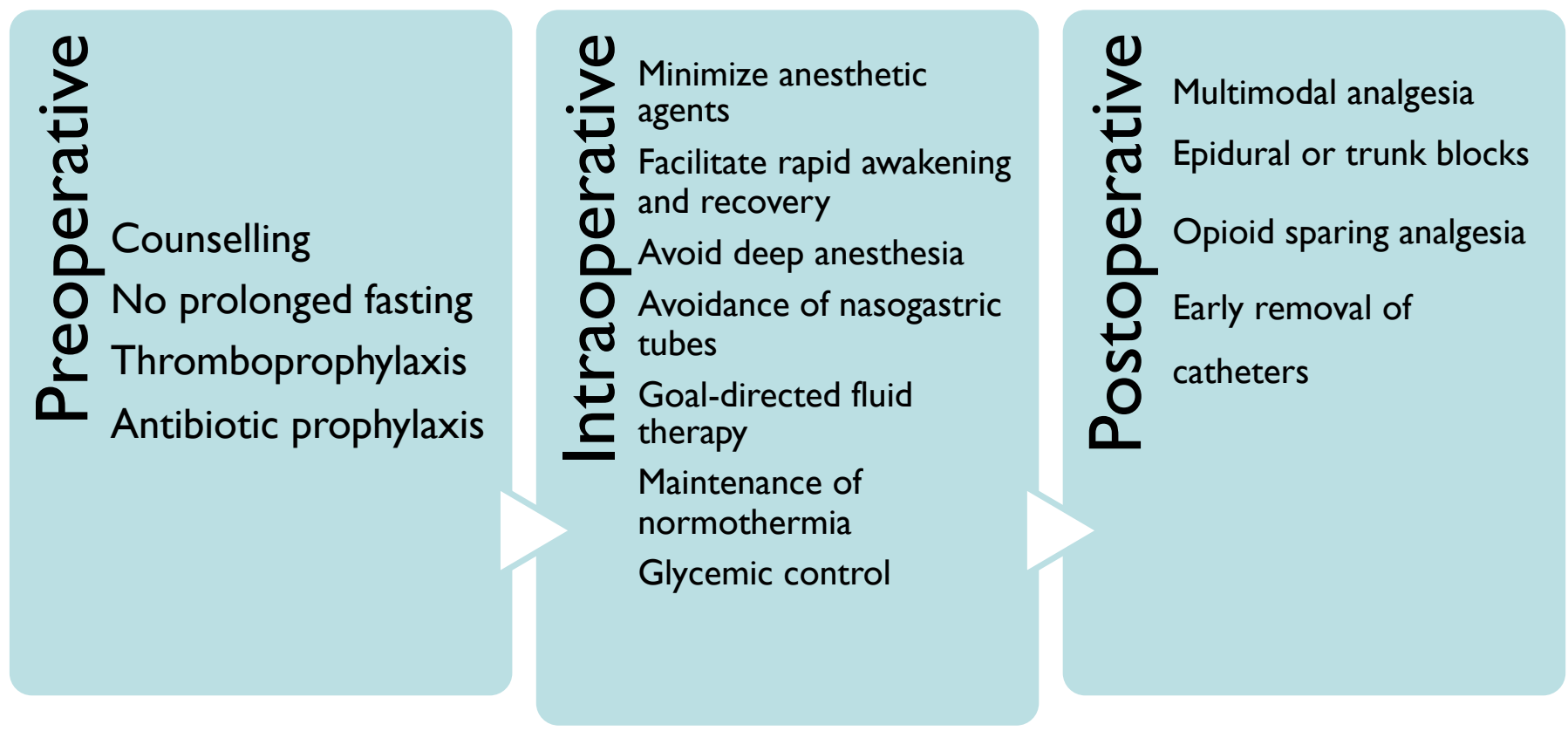

Figure 1. ERAS recommendations in terms of anesthesia in abdominal surgery
Preoperative sedatives were minimized to $50 \mu \mathrm{g}$ fentanyl and 1 mg midazolam. We administered $0.5 \%$ bupivacaine $5 \mathrm{ml}$ for right and $5 \mathrm{ml}$ for left rectus sheet blocks at T9 dermatomal level, $10 \mathrm{ml}$ for the right and $10 \mathrm{ml}$ for left subcostal TAP blocks.

Results: The surgery lasted for 35 minutes. A $10 \mathrm{~cm}$ incision was performed starting from $2 \mathrm{~cm}$ distal, up to $8 \mathrm{~cm}$ from the umbilicus (Figure 2). The patient did not need any additional sedation or analgesics during the surgery. No complications were observed during the block procedure and the surgery. The abdominal wall anesthesia provided 16 hours of postoperative analgesia and acetaminophen intravenous was continued thereafter. We assessed the pain by using numerical rating scale (NRS) between 0 and 10. The NRS scores reported by the patient at 1 st, 2 nd 6 th and 12 th postoperative hours were $0 / 10$. The patient was discharged after 24 hours.

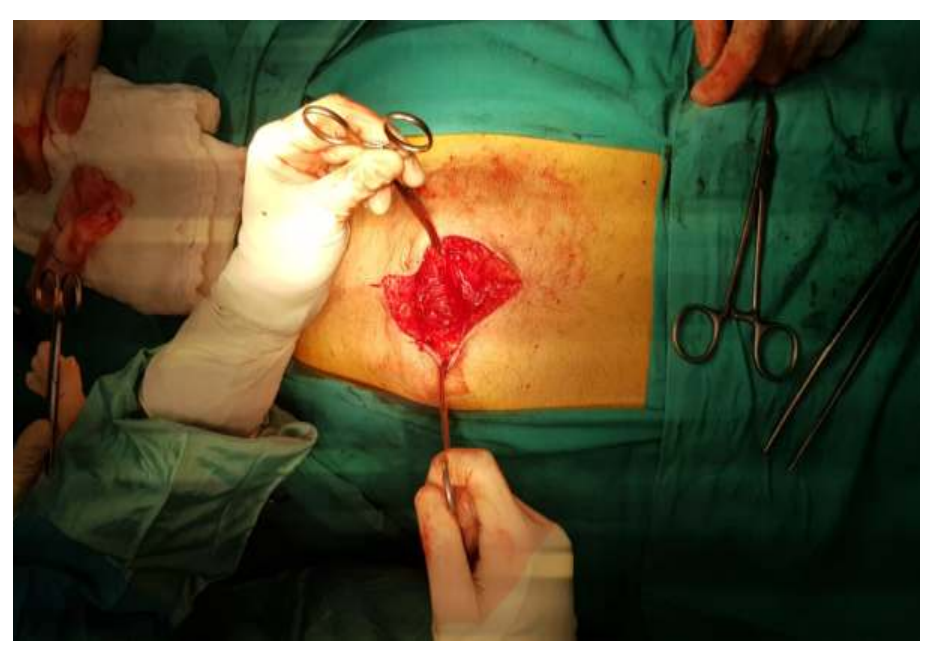

Figure 2. The midline incision performed for hernia repair.

Conclusions: Our case demonstrated that lesion targeted, ultrasonography guided abdominal wall anesthesia provided sufficient surgical anesthesia and postoperative analgesia in incisional hernia repair surgery. Early discharge and opioid-free postoperative analgesia were possible in our case with avoiding the risks of general anesthesia by using combination of bilateral rectus sheet and subcostal TAP blocks in accordance with ERAS protocols.

Feldheiser A. et al. Enhanced Recovery After Surgery (ERAS) for gastrointestinal surgery, part 2: consensus statement for anesthesia practice. Acta Anaesthesiol Scand. 2016;60:289-334 Simpson JC, Bao X, Agarwala A. Pain Management in Enhanced Recovery after Surgery (ERAS) Protocols. Clin Colon Rectal Surg. 2019; 32:121-128. Dunkman WJ, Manning MW. Enhanced Recovery After Surgery and Multimodal Strategies for Analgesia. Surg Clin North Am. 2018; 98:1171-1184 